\title{
Features involved in the diagnostic delay of cluster headache
}

\author{
J A van Vliet, P J E Eekers, J Haan, M D Ferrari, for the Dutch RUSSH study group
}

J Neurol Neurosurg Psychiatry 2003;74:1 123-1125

Background: Cluster headache $(\mathrm{CH})$ is a comparatively rare, very severe primary headache. Although circumscript and recognisable criteria are available, the diagnosis is often missed or delayed. Besides, while adequate and evidence based treatment is available in diagnosed cases, $\mathrm{CH}$ seems to be poorly managed. The authors performed a nationwide survey among $\mathrm{CH}$ patients, and looked for factors involved in the diagnostic delay.

Methods: The authors performed a nationwide mailing to all Dutch general practitioners (about 5800), and neurologists (about 560) and invited them to refer patients in whom the diagnosis $\mathrm{CH}$ was made or considered. Patients could also apply via the Dutch Headache Patients Society. A variety of clinical characteristics were assessed by means of questionnaires. Specifically, patients were asked about the time between their first episode and the diagnosis.

Results: The IHS criteria for $\mathrm{CH}$ were met by 1429 of 2001 responders, and 1163 of these filled in an extended questionnaire. The male to female ratio was 3.7:1. Mean age at onset was 32 (SD 14) years. Seventy three per cent had episodic $\mathrm{CH}, 21 \%$ had chronic $\mathrm{CH}$, and in $6 \%$ the periodicity was undetermined. The time between the first episode and the diagnosis ranged from 1 week to 48 years (median 3 years): $34 \%$ had consulted a dentist and $33 \%$ an ENT specialist before the diagnosis was established. Among factors that increased the diagnostic delay were the presence of photophobia or phonophobia, nausea, an episodic attack pattern and a low age at onset $(p<0.01)$. Sex or presence of restlessness during episodes did not influence the diagnostic delay.

Conclusion: $\mathrm{CH}$ remains unrecognised or misdiagnosed in many cases for many years. Photophobia or phonophobia and nausea were in part responsible for this delay, and should be recognised as part of the clinical spectrum of $\mathrm{CH}$. Many patients were first seen by a dentist or ENT specialist for their $\mathrm{CH}$ episodes, so more attention should be paid to educate first line physicians to recognise $\mathrm{CH}$, to improve the diagnostic process and so to expose patients to earlier and better treatment of $\mathrm{CH}$.

C luster headache $(\mathrm{CH})$ is a comparatively rare, severe primary headache. It is characterised by frequent episodes of very intense, unilateral pain in the orbital or temporal region. The episodes have a short duration, and are associated with one or more signs of autonomic dysfunction on the painful side.

Despite the well defined clinical description, it seems that the diagnosis is often missed or delayed in clinical practice, ${ }^{2}$ probably because of a limited knowledge of the disease and its clinical spectrum. Interictal headache or concomitant symptoms, such as photophobia or nausea during episodes, may distract from the appropriate diagnosis. The aim of this study was to describe a variety of clinical features of $\mathrm{CH}$ in a large sample of patients in order to identify reasons for the diagnostic delay.

\section{METHODS \\ Subject recruitment}

All neurologists (about 560) and general practitioners (GPs, about 5800) in the Netherlands were approached by a nationwide mailing and were asked to invite patients with $\mathrm{CH}$ or resembling $\mathrm{CH}$ to join the study. Announcements including the criteria of $\mathrm{CH}$ and the aims of the study were published in several Dutch medical journals and newspapers. Patients were also invited to participate by an announcement in the monthly magazine of the Dutch Society for Headache Patients while they could also register on a Dutch $\mathrm{CH}$ web site.

To screen for $\mathrm{CH}$, we developed a postal questionnaire. In this study, we exclusively included patients who met all features that are part of the IHS criteria for $\mathrm{CH}$.

Subsequently, all responding patients who met these criteria were sent a second, more extensive questionnaire, containing questions about a variety of clinical features, including the time between their first episode to the moment the diagnosis was made.

\section{Statistical analysis}

Data are expressed as percentage, mean (SD) or median (range). We used Student's $t$ test or Mann-Whitney U test for comparisons of continuous data and $\chi^{2}$ test to compare categorical data. Linear regression analysis was used to test relations between two continuous variables. A p level of $<0.05$ was considered as statistically significant.

\section{RESULTS}

A total of 2001 subjects were enrolled. The IHS criteria for $\mathrm{CH}$ were met by 1429 patients according to the screening questionnaire. Of these, $1163(81 \%)$ returned the second, extensive questionnaire and the results of these patients are presented here.

Mean age was 46 years (SD 12 years). There were 913 men and 250 women (male to female ratio 3.7:1). Seventy five per cent reported to have had $\mathrm{CH}$ attacks in the previous year; $41 \%$ of these had their most recent attack within the past month. Seventy three per cent of the patients had episodic $\mathrm{CH}$, and $21 \%$ had chronic $\mathrm{CH}$, of which more than half were of the secondary chronic type. The periodicity of the remaining $6 \%$ was undetermined (for example, first episode, or recent onset of $\mathrm{CH}$ ). Mean age at onset was 32 years (SD 14 years), with a significant later onset in chronic patients (38 (SD 18) years) than in episodic patients (32 (SD 16) years), $\mathrm{p}<0.001$. There was no statistical significant difference of age at onset between men (32 (SD 13)) and women (33 (SD 16)).

Median attack duration was 80 minutes, and mean attack frequency was 3.3 per day (SD 2.2 per day). Mild, continuous headache between the attacks ("interictal headache") was reported by $14 \%$ of the episodic patients, and $28 \%$ of the 


\begin{tabular}{|c|c|c|c|c|}
\hline Clinical feature & $\begin{array}{l}\% \text { Of patients } \\
\text { with clinical } \\
\text { feature }\end{array}$ & $\begin{array}{l}\text { Diagnostic delay } \\
\text { when feature } \\
\text { present* }\end{array}$ & $\begin{array}{l}\text { Diagnostic delay } \\
\text { when feature not } \\
\text { present* }\end{array}$ & $\mathrm{p}$ Value \\
\hline Male sex & 79 & $3(\leqslant 1-45)$ & $3(\leqslant 1-48)$ & 0.448 \\
\hline Episodic $\mathrm{CH}$ & 73 & $3(\leqslant 1-48)$ & $1(\leqslant 1-28)$ & 0.001 \\
\hline Nausea during attacks & 27 & $4(\leqslant 1-45)$ & $2.3(\leqslant 1-48)$ & 0.001 \\
\hline Vomiting during attacks & 12 & $4.8(\leqslant 1-37)$ & $2.5(\leqslant 1-48)$ & 0.003 \\
\hline Photophobia or phonophobia & 54 & $3(\leqslant 1-48)$ & $2(\leqslant 1-48)$ & 0.022 \\
\hline Nocturnal onset of attacks & 78 & $3(\leqslant 1-48)$ & $2(\leqslant 1-35)$ & 0.009 \\
\hline Interictal headache & 16 & $2(\leqslant 1-42)$ & $3(\leqslant 1-48)$ & 0.078 \\
\hline Circadian rhythm & 64 & $3(\leqslant 1-48)$ & $2.5(\leqslant 1-40)$ & 0.459 \\
\hline Restlessness & 76 & $3(\leqslant 1-48)$ & $2(\leqslant 1-37)$ & 0.787 \\
\hline Pain radiating to jaw & 37 & $3(\leqslant 1-42)$ & $2.5(\leqslant 1-48)$ & 0.384 \\
\hline Alternating attack side & 11 & $6(\leqslant 1-34)$ & $2.5(\leqslant 1-48)$ & 0.001 \\
\hline
\end{tabular}

chronic patients $(p<0.001)$. Seventy eight per cent of the patients reported to have nocturnal onset of attacks, waking them up after a median time of 120 minutes sleep (range 15 minutes to 7 hours). Besides pain in the orbital or temporal area, $37 \%$ of the patients reported that the pain radiated to the lower jaw, upper jaw, or cheek.

The reported median time between the first attack and the diagnosis of $\mathrm{CH}$ was three years (range 1 week to 48 years). Factors that increased the time to diagnosis were an episodic pattern of attacks, presence of nausea, vomiting or photophobia and alternating attack sides (table 1). Furthermore, a lower age at onset was associated with a longer diagnostic delay $(p<0.01)$. There was no influence of sex on time to diagnosis.

The initial diagnosis was "cluster headache" in $22 \%$ of the cases, "sinusitis" in $21 \%$, "migraine" in $17 \%$, and "dental related" in $11 \%$. Sixteen per cent of the patients had made the diagnosis "cluster headache" themselves, by recognising $\mathrm{CH}$ symptoms described in articles in newspapers, books, etc. Dentists (34\%), ENT specialists (33\%), and alternative therapists $(33 \%)$ were equally often consulted for the headaches before diagnosis. Tooth extraction (16\%) and ENT operation $(12 \%)$ as a treatment for the headaches were reported.

\section{DISCUSSION}

In this study we found that the diagnosis of $\mathrm{CH}$ was delayed in many cases: a diagnostic delay of three years or more was found in half of the patients. Clinical features that seemed to increase the diagnostic delay were the presence of nausea, vomiting, and photophobia during $\mathrm{CH}$ attacks. As these symptoms are eminent in migraine, these patients were probably initially mistaken for migraine. However, these symptoms were comparatively frequently reported in this study and seem to be part of the clinical spectrum of $\mathrm{CH}$, as was reported previously. $^{34}$

In the IHS criteria for $\mathrm{CH}$ "strictly unilateral pain" is included. This may cause confusion, as the pain during an individual attack is unilateral but may, although rarely, switch sides in between attacks (usually in between cluster periods). Switching attack sides was reported in $11 \%$ of the cases in this study and these patients had an increased time to diagnosis. We therefore suggest that this feature may be included in future criteria of the IHS.

Another factor that was associated with an increased time to diagnosis was a low age at onset. The mean age at onset of $\mathrm{CH}$ is around 30 years, as our study confirms. $\mathrm{CH}$ in children is very rare, and often other diagnoses are considered first. ${ }^{5}$

Episodic patients had a longer diagnostic delay than chronic patients. Apparently, the episodic occurrence of the attacks, a very specific feature of $\mathrm{CH}$, does not seem to contribute to an early diagnosis. The long attack free periods between cluster periods in episodic cases could have postponed the diagnosis with several years, as episodic patients may be symptom free for consecutive months or years.

About one third of the patients were at some time in the diagnostic process referred to dentists or ENT specialists, or both. As was earlier recognised, ${ }^{2} \mathrm{CH}$ is comparatively often mistaken for sinusitis or a dental disorder. Increased knowledge of $\mathrm{CH}$ among referring first line physicians, but also ENT specialists and dentists, could improve recognition and treatment of $\mathrm{CH}^{6}{ }^{6}$ Unnecessary and invasive procedures such as tooth extraction or sinus operations, which were frequently reported, may consequently be avoided.

Sixteen per cent of the subjects, mostly chronic patients, reported a mild, continuous headache in between their $\mathrm{CH}$ attacks. This feature has, to our knowledge, not been described before in $\mathrm{CH}$. It can be caused by concomitant chronic daily headache, but it may also be an aspect of $\mathrm{CH}$ medication or $\mathrm{CH}$ pathophysiology.

A possible drawback of our study may be that we used questionnaires for collecting data. The decisive argument for us to apply this method was that in a study with large populations, a mail questionnaire is comparatively inexpensive and easy to use. Moreover, many patients may be unable to visit the hospital for a clinical interview but are able to cooperate in a questionnaire study. The use of postal questionnaires, however, has methodological disadvantages: there is no flexibility to rephrase questions or introduce examples so that the investigator can ensure that the responder understands what information is required. ${ }^{7}$ Furthermore, patients are thought to most accurately recall recent or most severe headaches. In our study, $75 \%$ of the patients reported to have had attacks in the previous year. Most patients were therefore probably able to accurately recall the details of their headaches. But as the severity and frequency of $\mathrm{CH}$ attacks might be varying in time, it is possible that duration and frequency of attacks were overestimated in this study. In addition, the time of the diagnostic delay could not exactly be discovered, as this had to be recollected from many years in some patients. This sort of bias cannot be prevented using other methods such as clinical interviews. Diagnosing primary headache syndromes is in any case based on retrospective data, provided by the patient. A prospective study could reveal more conclusive data on this issue.

Advantages of this study are the large number of patients and the coverage of the Netherlands. In this way, not only clinical patients were approached, but also those who were never seen by a specialist. Patients with milder forms of $\mathrm{CH}$, or patients with longlasting remission periods were therefore more likely to be included, giving a more representative view on $\mathrm{CH}$. Besides, we expect that strong attention was drawn on $\mathrm{CH}$ by our nationwide mailing to GPs and neurologists, which has possibly lead to more accurate diagnosis in some patients. 


\section{Authors' affiliations}

J A van Vliet, J Haan, M D Ferrari, Department of Neurology, Leiden University Medical Centre, Leiden, Netherlands

P J E Eekers, Department of Neurology, Het Spittaal, Zutphen,

Netherlands

J Haan, Department of Neurology, Rijnland Hospital, Leiderdorp,

Netherlands

Competing interests: none declared.

Correspondence to: Dr J Haan, Department of Neurology, K5Q, Leiden University Medical Centre, PO Box 9600, 2300 RC Leiden, Netherlands; jhaan@rijnland.nl

Received 16 January 2003

Accepted in revised form 4 March 2003

\section{REFERENCES}

1 Headache Classification Committee of the International Headache Society. Classification and diagnostic criteria for headache disorders, cranial neuralgias and facial pain. Cephalalgia 1988; suppl 7:1-96.

2 Klapper JA, Klapper A, Voss T. The misdiagnosis of cluster headache: a non-clinic, population based, internet survey. Headache 2000;40:730-5.

3 Nappi G, Micieli G, Cavallini A, et al. Accompanying symptoms of cluster attacks: their relevance to the diagnostic criteria. Cephalalgia 1992;12:165-8.

4 Bahra A, May A, Goadsby PJ. Cluster headache. A prospective clinical study with diagnostic implications. Neurology 2002;58:354-61.

5 Lampl C. Childhood-onset cluster headache. Pediatr Neurol 2002;27: 138-40.

6 Cady RK, Schreiber CP. Sinus headache or migraine? Considerations in making a differential diagnosis. Neurology 2002;58 (suppl 6):S10-14.

7 Lipton RB, Stewart WF, Solomon S. Questionnaire versus clinical interview in the diagnosis of headache. Headache 1992;32:55-6.

\section{Applications are invited for the post of Editor}

Journal of Neurology Neurosurgery and Psychiatry

JWWP is one of the premier journals in neurology. We now seek an editor who can increase its scientific and intemational reputation while maintaining its relevance to practising neurologists worldwide.

Applications from specialists in any branch of neurology. neurosurgery or psychlatry are inwited. Applications from outside the UK are encouraged and joint applications from two or more individuals willing to act as a team are welcomed.

Full editorial support and training will be provided. The journal will fund any necessary locum requirements. The successful applicantis) will be free to select hisher own team of associate editors and board members.

Closing date for applications is 1st August 2003. Interviews will be held on 9 th September 2003. It is envisaged that the outgoing editor will gradually hand over during the fourth quarter of 2003 and the successful candidate will otficially take up the post of editor in January 2004.

Further details of the post can be discussed with the publishing director, Mrs Alex Williamson and/or the retiring editor Professor Christopher Kennard (Tel +44 (0)20 8846 7598; emsil c.kennardeic.acuk». A job description is available on request from Mrs Williamson.

Applications should be sent to Mrs Alex Williamson, BMJ Publishing Group, BMA House, Tavistock Square, London WC1H 9IR, UK. Tel +44 (0)20 7383 6069/6169; Fax +44 (0)20 7383 6668; email awilliamsonebmigroup.com

$\mathrm{BMJ}$ 